\title{
La adopción de las Normas Internacionales de Información Financiera en Colombia
}

\begin{abstract}
This article is aimed at describing an overview of the adoption of International Financial Reporting Standards in Colombia. For our study we used the methodology based on desk research. Throughout this article, we will show an accounting analysis about the Colombian normativity, the evolution of these standards and how they work in SMEs, until getting to the convergence period.
\end{abstract}

Keywords: IFRS, IAS, SMEs.

\section{RÉSUMÉ}

Ce document présente un aperçu général de l'adoption des normes internationales d'information financière en Colombie. Pour la réalisation de cette étude, nous avons fait des recherches documentaires. Nous montrerons tout au long de ce document une analyse comptable de la normativité colombienne, l'évolution de ces normes et leur utilisation dans les PMEs jusqu'à la période de convergence.

Mots-clés: NIIF, NCI, PMEs.

\section{Adopting International Financial Reporting Standards in Colombia \\ L'adoption des Normes Internationales d'Information Financière en Colombie}

ROCÍO VERGARA-DE LA OSSA

Fundación Tecnológica Antonio de Arévalo - TECNAR

Colombia

rocio.vergara@tecnar.edu.co

CLAUDIA INES LONDOÑO-VEGA

Fundación Tecnológica Antonio de Arévalo - TECNAR

Colombia

claudia.londono@tecnar.edu.co

NAZLY DEL CARMEN PÉREZ-BENÍTEZ

Fundación Tecnológica Antonio de Arévalo - TECNAR

Colombia

nazly.perez@tecnar.edu.co

ROBERTO TORRES-CASTELLAR

Fundación Tecnológica Antonio de Arévalo - TECNAR

Colombia

roberto.torres@tecnar.edu.co

\section{RESUMEN}

El presente documento tiene por objetivo describir el panorama general de la adopción de las normas internacionales de información financiera en Colombia ad portas de su implementación. La metodología empleada es la investigación documental. Durante el recorrido del artículo, se revisa la normatividad colombiana en materia contable, la evolución de las normas internacionales de información financiera, las normas internacionales de información financiera para Pymes, hasta llegar al periodo de convergencia.

Palabras clave: NIIF, NIC, Pymes.

Clasificación JEL: L10, L15, F30, G15, G18. 


\section{INTRODUCCIÓN}

Desde el año 2004, son muchas las naciones que han venido convergiendo en una $\mathrm{u}$ otra manera, hacia las NIIF, ejemplo de ellas tenemos, países de la Unión Europea, Centroamérica, Suramérica y de algunos estados de Asia y Oceanía. Por otro lado de las organizaciones que iniciaron con su aplicación están las pertenecientes al mercado de valores, empresas de interés público, entidades financieras y de seguros, empresas de servicios públicos, entre otras.

Las normas internacionales de contabilidad cuando se apliquen en Colombia podrían traer beneficios o debilidades en el entorno empresarial. Colombia, debe aplicar, incurrir y regir los parámetros específicos de acuerdo a la Ley 1314 el 13 de julio de 2009. Teniendo en cuenta la ley mencionada, se discutió y decidió la mejor forma de constituir El Consejo Técnico de Contaduría Pública quien será el órgano par en Colombia del International Accounting Standards Board (IASB), y que estará en contacto con este organismo internacional en la convergencia a las normas internacionales de información financiera para pequeñas y medianas empresas (NIIF para Pymes).

Debido a lo anterior es necesario llevar a cabo esta implementación de la manera más adecuada y a los ajustes que cada pequeña y mediana empresa (Pyme) requiera, teniendo en cuenta sus dimensiones al interior de la compañía con el fin de tomar buenas decisiones que reflejaran la solidez y permanencia en el desarrollo del impacto que genera este costo durante el proceso de transición y su convergencia.

\section{ORIGEN DE LAS NIIF}

El análisis histórico de la contabilidad nos permite conocer el pasado el presente y visualizar el futuro de la contabilidad. Desde los primitivos sistemas de escritura, el hombre los ha utilizado para llevar a cabo el registro de aquellos datos de la vida económica que le era preciso recordar, pero es en 1.494 cuando se rompe en dos la historia de la Contabilidad con la aparición de la partida doble y su divulgación por Lucas Paccioli.

A partir de 1495 hasta nuestros días la contabilidad se divide en tres periodos, el primero de 1494 hasta 1794 periodo en el cual se desarrollan los principios de la partida doble. El segundo desde 1975 hasta 1930 en donde aparecen escuelas y doctrinas contables. Y el tercero desde 1931 hasta hoy el cual se caracteriza por la formación de los principios contables y los estándares internacionales de contabilidad.

Según Tua (2004) el comienzo del periodo científico de la contabilidad se inicia en 1.846 con la escuela de Lombarda De Francesco Villa cuando esta separa la teneduría de libros de la verdadera ciencia contable. Posteriormente en 1.867 aparece la escuela Toscana liderada por Marchi, Cerbon y Rossi y en la cual se establecen los usuarios interesados en la vida de la Empresa. Así como la presentación de un sistema contable basado en cuatro libros. Y en 1.891 surge la escuela veneciana liderada por Fabio Besta y plantea un enfoque económico de la contabilidad patrimonial

El pionero en darle a la contabilidad un carácter de disciplina formal según Montesinos (1994) fue Eugene de Fages de Latour en 1924 y este enfoque se consolida con Richard Mattessich cuando introdujo la formulación matricial de los sistemas con- 
tables. En 1926, Schanalenbach presenta la primera propuesta formal de un plan de cuentas bien elaborado, más tarde da lugar al plan contable alemán. Schneider por su parte hizo grandes aportes a la contabilidad de costos con su obra Contabilidad Industrial.

El tercer periodo se inicia desde 1931 hasta nuestros días se origina con la gran depresión de los años 30 y se caracteriza por dos tendencias del método de investigación contable. La primera tendencia de investigación contable positiva influenciada por la escuela positivista comtiana y el pragmatismo anglosajón que desarrolla los principios de contabilidad generalmente aceptados, en Norteamérica estos fueron denominados US GAAP en los demás países tomo el nombre del país seguido de la terminación GAAP (Ramírez, 2014).

La segunda tendencia se caracteriza por el carácter científico de la contabilidad y de la investigación contable en donde se da el establecimiento de un modelo que remarcaba la capacidad de generar beneficios, al cual le siguió el programa de establecimiento de normas ASC en Reino Unido (Ibíd.). Otros autores eran partidarios de un enfoque basado en la utilidad de la decisión, lo cual conducía a determinar cómo los objetivos de los Estados Financieros constituían el punto de partida para la investigación contable financiera.

Tua (2009) simplifica en dos grandes grupos los diferentes sistemas contables adoptados a lo largo de la historia. Por un lado, los sistemas contables anglosajones, especialmente EEUU y Reino Unido, tienen el objetivo prioritario de presentar la información para la correcta toma de decisiones por parte de los inversores y los analistas. Normalmente nacidos en el seno de países que históricamente han tenido un tempra- no desarrollo económico donde uno de sus pilares fundamentales han sido unos poderosos mercados de capitales, con un alto grado de eficiencia.

El mundo occidental ha apostado por una contabilidad orientada al suministro de información útil para la toma de decisiones económicas, en las que prima, por encima de cualquier otra consideración, el enfoque predictivo, todo ello con el punto de mira puesto en el mercado de capitales. Y ello es así, sin duda, por el trascendental papel que tales mercados desempeñan en la actividad económica. Buena muestra de este tránsito lo constituyen las Normas Internacionales de Contabilidad, claramente orientadas a la predicción, en la medida en que están destinadas a regular la información que se vierte a los mercados de capitales.

La influencia de estos sistemas contables en el marco conceptual de las normas internacionales de contabilidad es evidente. Según Zeff (2012) "en su diseño del marco conceptual en 1989 el antiguo IASC recorría el mismo camino que los Standard setters anglosajones habían recorrido años atrás". Así, se orientó hacia un enfoque de balance basado en el valor de los activos y pasivos, muy alejado del tradicional enfoque basado en la cuenta de resultados consistente en la correcta distribución temporal de los flujos de caja de acuerdo con su devengo contable. Así se comenzó a considerar la valoración a valor razonable de los activos y pasivos por encima del tradicional criterio del costo histórico.

Por otro lado, los sistemas contables de control tradicionalmente desarrollados en países latinos, cuya prioridad es suministrar la información requerida por propietarios y acreedores, con el objetivo de controlar la gestión y rendir cuentas. Se trata de sis- 
temas propios de países con un desarrollo económico más tardío. Con fuentes de financiación menos dependientes de los mercados bursátiles (Tua, 2009).

Las NIIF o IFRS hacen referencia las Normas Internacionales de Información Financiera o en ingles Internacional Financial Reporting Standard, las cuales fueron emitidas por la IASB (Internacional Accounting Estándar Board) o Junta de Normas Internacionales de Contabilidad; con el objetivo de crear un estándar que permitiera la comparabilidad de la información financiera. Estas buscan dar una estructura uniforme a la información financiera y a la contabilidad de las empresas, lo que se quiere es que a corto tiempo sea el lenguaje universal.

\section{¿QUÉ SON LAS NIIF?}

Las NIIF son consideradas un conjunto de normas en el sentido de que establecen normas generales, así como dictar tratamientos específicos. Las Normas Internacionales de Información Financiera comprenden:

- Normas Internacionales de Información Financiera (Normas después de 2001).

- Normas Internacionales de Contabilidad (Normas antes de 2001).

- Interpretaciones NIIF (Normas después de 2001) CINIIF.

- Interpretaciones de las NIC (Normas antes de 2001) SIC.

\section{Acrónimos:}

NIIF: Normas Internacionales de Información Financiera.

IFRS: International Financial Reporting Standards (nuevas normas que iniciaron su secuencia en el años 2003).

IASB: Junta de Normas Internacionales de Contabilidad.

IAS: International Accouting Standard (Normas Internacionales de Contabilidad) Son las normas emitidas previamente cuya secuencia fue del número 1 al 41.

IFRIC: International Financial Reporting Interpretation Commitee (Comité Internacional de Reportes Financieros) Son las interpretaciones actuales; no todas las NIC/ IAS y las SIC están vigentes.

SIC: Interpretaciones anteriores cuya secuencia llegó hasta el número 32.

Las IFRS fueron adoptadas por primera vez en el año 2005 en 92 países. Todas las compañías registradas en Bolsa de Valores de los 25 países miembros de la Unión Europea están preparando estados financieros bajo IFRS, a partir del 1 de enero del 2005.

Son en total 13 NIIF-IFRS y 29 NIC - IAS, NIIF para Pymes 1, Interpretación de NIIF-IFRIC 17 y 8 Interpretaciones NIC-SIC.

Tabla 1. Tipos de NIFF/IFRS

\begin{tabular}{|c|c|c|c|}
\hline Norma & Descripción & Revisada & Vigencia \\
\hline NIC/IAS 1: & $\begin{array}{c}\text { Presentación de estados } \\
\text { financieros }\end{array}$ & 1997 & \\
\hline NIC/IAS 2: & Inventarios & diciembre de 2003 & 1 de Enero de 2005 \\
\hline NIC/IAS 7: & Estado de flujos de efectivo & Diciembre de 1992 & 1 de Enero de 1994 \\
\hline NIC/IAS 8: & $\begin{array}{c}\text { Políticas contables, cambios en } \\
\text { estimados contables y errores }\end{array}$ & diciembre de 2003 & 1 de Enero de 2005 \\
\hline
\end{tabular}


ROCÍO VERGARA-DE LA OSSA, CLAUDIA INÉS LONDOÑO-VEGA, NAZLY DEL CARMEN PÉREZ-BENÍTEZ, ROBERTO TORRES-CASTELLAR La adopción de las Normas Internacionales de Información Financiera en Colombia

\begin{tabular}{|c|c|c|c|}
\hline Norma & Descripción & Revisada & Vigencia \\
\hline NIC/IAS 10: & $\begin{array}{l}\text { Hechos posteriores a la fecha del } \\
\text { balance }\end{array}$ & diciembre de 2003 & 1 de Enero de 2005 \\
\hline NIC/IAS 11: & Contratos de construcción & Diciembre de 1993 & 1 de Enero de 1995 \\
\hline NIC/IAS 12: & Impuesto a las ganancias & Octubre de 2000 & 1 de Enero de 2001 \\
\hline NIC/IAS 14: & $\begin{array}{l}\text { Información financiera por } \\
\text { segmentos }\end{array}$ & 1997 & 1 de Enero de 2001 \\
\hline NIC/IAS 16: & Propiedades, planta y equipo & 2003 & 1 de enero de 2005 \\
\hline NIC/IAS 17: & Arrendamientos & diciembre de 2003 & 1 de enero de 2005 \\
\hline NIC/IAS 18: & Ingresos & Diciembre del 1998 & 1 de enero del 2001 \\
\hline NIC/IAS 19: & Beneficios a empleados & 2000 & 1 enero del 2001 \\
\hline NIC/IAS 20: & $\begin{array}{c}\text { Contabilización de las } \\
\text { subvenciones oficiales e } \\
\text { información a revelar sobre } \\
\text { ayudas públicas }\end{array}$ & $\begin{array}{l}\text { reordenada: } 1 \text { enero } \\
\text { de } 1994\end{array}$ & 1 de Enero de 1984 \\
\hline NIC/IAS 21: & $\begin{array}{c}\text { Efectos de las variaciones en las } \\
\text { tasas de cambio de la moneda } \\
\text { extranjera }\end{array}$ & Diciembre de 1993 & 1 de enero de 1995 \\
\hline NIC/IAS 23: & Costos por intereses & (revisada en 1993) & 1 enero de 1995 \\
\hline NIC/IAS 24: & $\begin{array}{c}\text { Información a revelar sobre } \\
\text { partes relacionadas }\end{array}$ & $\begin{array}{c}\text { Reordenada: } \\
\text { Diciembre del } 2003\end{array}$ & 1 enero del 2005 \\
\hline NIC/IAS 26: & $\begin{array}{c}\text { Contabilización e información } \\
\text { financiera sobre planes de } \\
\text { beneficio por retiro }\end{array}$ & reordenada en 1994 & 1 enero de 1988 \\
\hline NIC/IAS 27: & $\begin{array}{l}\text { Estados financieros consolidados } \\
\text { y contabilización de inversiones } \\
\text { en subsidiarías }\end{array}$ & $\begin{array}{l}\text { (reordenada en } 1994 \\
\text { y diciembre de 2003) }\end{array}$ & 1 enero de 1990 \\
\hline NIC/IAS 28: & $\begin{array}{c}\text { Contabilización de inversiones } \\
\text { en empresas asociadas }\end{array}$ & diciembre de 2003 & 1 enero de 2005 \\
\hline NIC/IAS 29: & $\begin{array}{c}\text { Información financiera en } \\
\text { economías hiperinflacionarias }\end{array}$ & Reordenada en 1994 & 1enero de 1990 \\
\hline NIC/IAS 30: & $\begin{array}{l}\text { Información a revelar en los } \\
\text { estados financieros de bancos y } \\
\text { entidades financieras similares }\end{array}$ & Reordenada en 1994 & 1 enero de 1991 \\
\hline NIC/IAS 31: & $\begin{array}{l}\text { Información financiera de los } \\
\text { intereses en negocios conjuntos }\end{array}$ & Diciembre de 2003 & 1 enero de 2005 \\
\hline NIC/IAS 32: & $\begin{array}{c}\text { Instrumentos financieros; } \\
\text { presentación e información a } \\
\text { revelar }\end{array}$ & 1998 y 2003 & 1 enero de $1996 / 2005$ \\
\hline NIC/IAS 33: & Ganancias por acción & (Revisada en 1997) & 1 enero de 1999 \\
\hline NIC/IAS 34: & Estados financieros intermedios & Junio de 1998 & 1 junio de 1999 \\
\hline NIC/IAS 36: & Deterioro del valor de los activos & junio 1998 & 1 julio de 1999 \\
\hline NIC/IAS 37: & $\begin{array}{l}\text { Provisiones, activos contingentes } \\
\text { y pasivos contingentes }\end{array}$ & 1998 & 1 julio de 1999 \\
\hline NIC/IAS 38: & Activos intangibles & septiembre de 1998 & 1 julio de 1999 \\
\hline NIC/IAS 39: & $\begin{array}{l}\text { Instrumentos financieros: } \\
\text { reconocimiento y medición }\end{array}$ & $\begin{array}{c}\text { octubre } 2000 \text { y } \\
\text { diciembre del } 2005\end{array}$ & $\begin{array}{c}\text { Revisada en octubre } \\
2000 \\
\end{array}$ \\
\hline NIC/IAS 40: & Propiedades de inversión & abril de 2000 & 1 enero de 2001 \\
\hline NIC/IAS 41: & Agricultura & diciembre de 2000 & 1 enero de 2003 \\
\hline
\end{tabular}




\begin{tabular}{|c|c|c|c|}
\hline Norma & Descripción & Revisada & Vigencia \\
\hline \multicolumn{4}{|c|}{ Interpretaciones Del Comité Permanente. } \\
\hline NIIF/IFRS 1: & $\begin{array}{l}\text { Adopción por primera vez de las } \\
\text { NIIF/IFRS }\end{array}$ & 19 junio de 2003 & \\
\hline NIIF/IFRS 2: & $\begin{array}{c}\text { Compensación basada en } \\
\text { acciones }\end{array}$ & febrero de 2004 & \\
\hline NIIF/IFRS 3: & Combinaciones de negocios & marzo de 2004 & \\
\hline NIIF/IFRS 4: & Contratos de seguros & marzo de 2004 & \\
\hline NIIF/IFRS 5: & $\begin{array}{c}\text { Activos no corrientes disponibles } \\
\text { para la venta y operaciones } \\
\text { descontinuadas }\end{array}$ & marzo de 2004 & \\
\hline NIIF/IFRS 6: & \multicolumn{3}{|c|}{ Exploración y explotación de productos minerales. } \\
\hline NIIF/IFRS 7: & \multicolumn{3}{|c|}{ Revelación de instrumentos financieros } \\
\hline IFRIC 1: & \multicolumn{3}{|c|}{ Cambios en pasivos por desmantelamiento, restauración y similares. } \\
\hline IFRIC 2: & \multicolumn{3}{|c|}{$\begin{array}{l}\text { Contabilización de las participaciones en cooperativas e instrumentos de } \\
\text { participación similares. }\end{array}$} \\
\hline IFRIC 4: & \multicolumn{3}{|c|}{ Identificación de arrendamientos contenidos en algunos contratos. } \\
\hline IFRIC 5: & \multicolumn{3}{|c|}{$\begin{array}{l}\text { Derechos en fondos creados para desmantelamiento, restauración o } \\
\text { rehabilitación ambiental. }\end{array}$} \\
\hline SIC-7: & \multicolumn{3}{|c|}{ Introducción del euro } \\
\hline SIC-10: & \multicolumn{3}{|c|}{ Asistencia del gobierno sin relación específica con actividades de explotación } \\
\hline SIC-12: & \multicolumn{3}{|c|}{ Consolidación de entidades de propósito especial } \\
\hline SIC-13: & \multicolumn{3}{|c|}{$\begin{array}{l}\text { Entidades controladas conjuntamente -Aportaciones no monetarias (en } \\
\text { especie) de los partícipes }\end{array}$} \\
\hline SIC-15: & \multicolumn{3}{|c|}{ Arrendamientos operativos- Incentivos } \\
\hline SIC-21: & \multicolumn{3}{|c|}{ Impuesto sobre las ganancias- Recuperación de un activo revaluado } \\
\hline SIC-25: & \multicolumn{3}{|c|}{$\begin{array}{l}\text { Impuesto sobre las ganancias - Cambios en el estatus de la sociedad o sus } \\
\text { accionistas o socios }\end{array}$} \\
\hline SIC-27: & \multicolumn{3}{|c|}{$\begin{array}{l}\text { Evaluación del fondo económico de las transacciones que adoptan la forma } \\
\text { legal de un arrendamiento operativo. }\end{array}$} \\
\hline SIC-29: & \multicolumn{3}{|c|}{ Información a revelar-Acuerdos de concesión de servicios } \\
\hline SIC-31: & \multicolumn{3}{|c|}{ Ingresos ordinarios - Permutas que comprenden servicios de publicidad } \\
\hline SIC-32: & \multicolumn{3}{|c|}{ Activos intangibles - Desarrollo de sitios en Internet } \\
\hline
\end{tabular}

Tomada de: Actualicese.com (2005).

La simplificación de las NIIF Pymes se debió a que el ente regulador vio la necesidad de orientar la norma hacia las necesidades de usuarios de empresas no cotizadas y cuya financiación difiere de las empresas cotizadas, sin embargo las decisiones de los financiadores son semejantes pues ambos evalúan la capacidad del ente económico para generar flujos de efectivo adicional las Pymes cuentan con unos recursos más limitados lo cual conllevo a replantear una simplificación de los requisitos de la infor- mación teniendo en cuenta la relación costo-beneficio. ACFÍ Auditores, (Contadores y Consultores Financieros, s.f).

El consejo de Normas Internacionales define a las normas internacionales como normas contables adaptadas por el IASB, que constituyen los estándares internacionales o normas internacionales en el desarrollo de la actividad contable en las cuales se establecen los lineamientos para llevar la contabilidad de la forma como es aceptable 
a nivel mundial. Las NIIF son las estructura básica de la contabilidad internacional que propicia a los usuarios información relevante, comprensible y consistente. ACFÍ Auditores (Ibíd.).

En el presente trabajo se definen las pequeñas y medianas entidades como aquellas que no tienen la obligación pública de rendir cuentas y publican estados financieros con propósito de información general para usuarios externos. Dentro de los temas tratados por las NIIF completas y omitidos en las NIIF Pymes tenemos:

- $\quad$ Las ganancias por acción NIC 33

- Información Financiera Intermedia NIC 34

- Información financiera por segmentos NIC 8

- Contabilización separada de los activos mantenidos para la venta NIC 5

Los temas incluidos en las NIIF Pymes que no se tratan en las NIIF completas tenemos:

- Conceptos y principios generales sección 2

- Estados Financieros combinados sección 9

- Emisión inicial de acciones u otros instrumentos de patrimonio sección 22

- Venta de opciones, derechos y certificados de opciones para compra de acciones sección 22

- Capitalización de ganancias o emisiones gratuitas y división de acciones sección 22

- Diferencias derivadas de simplificaciones en criterios de reconocimiento y medición, eliminación de opciones de políticas contables o reducción de exigencias de información (Ibíd.).

\section{¿Para quiénes son las NIIF?}

Esta información es importante difundir para los empresarios, accionistas, administradores, personal contable, auditores, usuarios de estados financieros y demás agentes interesados en su conocimiento y aplicación.

Los objetivos que se establece la IASB son "desarrollar estándares contables de calidad, compresibles y de cumplimiento forzoso, que requieran información de alta calidad, transparente y comparable dentro de los estados financieros para poder tomar decisiones en función de estos. Esto es muy importante que sea así y que se cumpla porque hay mucha gente detrás de esa información que depende de ella para su trabajo, como:

- Los propietarios de la empresa en primer lugar, para conocer la evolución del negocio y si este es rentable.

- La administración para conocer en que necesita mejorar y llevar a cabo la toma de decisiones en consecuencia.

- Los acreedores porque necesitan saber la liquidez de la empresa, y si puede cumplir con sus obligaciones.

- El estado debido a que debe recaudar sus impuestos en función de esos datos.

- Los contadores y auditores, para que puedan asesorar y evaluar correctamente a la empresa en donde trabajan

\section{¿Qué países están sujetos a las NIIF?}

De acuerdo a la ACFI (s.f):

En la actualidad son ya muchos los países que se han sumado a la aplicación de las NIIF para sus empresas independientemente del tamaño de sus empresas, porque según los expertos estas han sido 
diseñadas para grandes empresas y multinacionales. Entre ellos están los veinticinco países miembro de la UE, y en América: Colombia, México, Estados Unidos, Costa Rica, Ecuador, Guatemala, Honduras, Panamá, Perú y República Dominicana. Otros como Japón, Singapur, Australia y en Febrero China también se unieron.

\section{Antecedentes a la implementación de las NIC y NIIF en Colombia}

En Colombia la práctica Contable está regulada por el Gobierno Nacional, esta se inició con la legislación mercantil al imponer al comerciante la obligación de organizar su contabilidad, y se le prescribieron pautas sobre el contenido y formas de llevar los libros de contabilidad para lo cual se emitieron leyes y decretos hasta llegar a los principios y normas de contabilidad generalmente aceptados los cuales se encuentran enmarcados en los Decretos 2649 y 2650 de 1993, el Código de Comercio, Ley 145 de 1960, Ley 43 de 1990.

A través de los tiempos han sufrido modificaciones a través de organismos de control y vigilancia, lo cual nos indica que los cambios han sido reiterados pero sin llegar a alcanzar los niveles de exigencia y competitividad enmarcados en las Normas Internacionales de Contabilidad (NIC), el gobierno siendo un conocedor de este hecho lo manifiesta a través del artículo 63 de la ley 550 de 1999, en la actualidad artículo 122, inciso 1 de la ley 1116 de Diciembre del 2006.

La globalización, los tratados de libre comercio que se están suscribiendo por nuestro país, la adopción del uso de las normas NIC y NIIF por más de 120 países, la necesidad de construir la información financiera en el lenguaje de los negocios así como el de generar espacio de confianza y trazabilidad de las operaciones trajo consigo una verificación de las normas contables internas que se tienen en nuestro país y a replantear la aplicación de las normas establecidas por la Junta de Estándares Internacionales de Contabilidad, con la visión de armonizar la información financiera a nivel mundial eliminando barreras, implementando modelos que responden a los criterios de comparabilidad.

Este proceso de verificación y análisis de las condiciones particulares de la realidad en nuestro país que ha entrado en una globalización y apertura mundial a través de tratados, convenios y acuerdos a nivel mundial que lo impulsan presentar unos estados financieros acordes a el ámbito internacional, consideradas de alta calidad presentando una imagen fidedigna, real y oportuna basadas en las normas emitidas por Internacional Accounting Standard Board (Anteriormente denominado Internacional Accounting Standard Committe).

Y se emana la Ley 1314 de 2009, Por la cual se regulan los principios y normas de contabilidad e información financiera y de aseguramiento de información aceptados en Colombia, se señalan las autoridades competentes, el procedimiento para su expedición y se determinan las entidades responsables de vigilar su cumplimiento. Por último se emite Decreto 3022 del 3013 NIIF para Pymes dando un margen de convergencia para el 2014 y para las Pymes el 2015, siendo la presentación de la información contable en el 2014 bajo los dos lineamientos.

Con el proyecto de ley 550 de 1999 en su artículo 63 el gobierno colombiano se pronuncia ante la necesidad de ajustar la 
normatividad contable colombiana en las áreas de auditoria, revisoría fiscal y presentación de la información financiera.

Esta situación genera varios pronunciamientos por cuanto se estaría transformando totalmente la práctica contable, ocasionando posiciones encontradas por diferentes profesionales, hay quienes están a favor y en contra, se trae a colación el concepto de Blanco (1997), Presidente del Instituto Nacional de Contadores Públicos en Colombia; quien afirma: "El modelo contable Colombiano que se utiliza actualmente se adoptó en mil novecientos noventa y tres (1993) con la expedición del decreto 2649; fue un modelo bastante armónico con el marco conceptual y las normas internacionales de contabilidad vigentes en ese momento, ya que los tomó como patrón".

Una opinión diferente es emitida por Franco (2002), Ex - presidente de la Federación Colegio Colombiano de Contadores Públicos, quien presenta posición en contrario frente a la implementación de las Normas Internacionales y argumenta: "las únicas empresas que requieren informes estandarizados son las que están incrustadas en el comercio internacional y estas no representan el 5\% de las Colombianas; no se puede sacrificar a toda una comunidad a transformar sus prácticas contables".

Esto nos implica un cambio de cultura tanto de la profesión contable como a nivel empresarial, incorporando esta práctica contable en aquellos principios que difieren con las Normas Contables Colombianas, analizar y unificar criterios y estableciendo modelos de adopción de una forma cronometrada y controlada de tal manera que no se traumatice el normal desarrollo de su actividad económica.
En Colombia se da la adopción de las Normas internacionales de Información Financiera NIIF, de manera paulatina tomando como base el año de 1973 en el cual nace el Comité de Normas Internacionales de Contabilidad (IASC), como resultado de un acuerdo tomado por ciertos organismos a nivel mundial (Alemania, Australia, Canadá, Estados Unidos, Francia, Holanda, Japón, México, Irlanda e Inglaterra).

Con base en la constitución de este organismo, se estableció un esquema normativo a nivel internacional llamado Normas Internacionales de Contabilidad (NIC), el cual se conformó de los siguientes pronunciamientos:

a) Normas Internacionales de Contabilidad, NIC (International Accounting Standards IS), emitidas por el Comité de Normas Internacionales de Contabilidad (International Accounting Standards Comittee, IASC).

b) Interpretaciones de las Normas Internacionales de Contabilidad, emitidas por el Comité de Interpretaciones de IASC (Standing Intterpretations Commitée, SIC).

El Consejo de Vigilancia de la Contaduría Pública y Auditoría, con fecha 2 de septiembre de 1999, tomó acuerdo, que en su segundo párrafo literalmente dice: En la preparación de los estados financieros deberán usarse Normas Internacionales de Contabilidad dictadas por el Comité de normas internacionales de Contabilidad (IASC) y/o aquellas regulaciones establecidas por este Consejo.

Pero se tenía en cuenta que aún se encontraban vigentes las disposiciones del Código de Comercio, que en sus artículos 443 y 444 establecían cuales deberían de ser las partidas que conformaban los estados financieros. 
La norma Colombiana inicia prácticamente el 30 de diciembre cuando el Ministerio de Comercio, Industria y Turismo de Colombia expide el Decreto 4946 de 30 de diciembre de 2011, sobre las Normas Internacionales de Información Financiera (NIIF), por el cual se dictan disposiciones en materia del ejercicio de aplicación voluntaria de las normas internacionales de contabilidad e información financiera.

Es así como en el año 2010 se inició el proceso de reglamentación y se conformó el CTCP con 4 miembros para establecer el plan de direccionamiento creando para tal fin los comités técnicos ad-honorem del sector financiero y el sector real. El 22 de junio de 2011 el Consejo Técnico de la Contaduría Pública, emite el documento final en cumplimiento de su función, presentó al Gobierno Nacional el Direccionamiento Estratégico del proceso de convergencia de las normas de contabilidad e información financiera y de aseguramiento de la información, con estándares internacionales.

En dicho direccionamiento estratégico el Consejo Técnico de la Contaduría Pública le recomendó al Gobierno Nacional que el proceso de convergencia a estándares internacionales de contabilidad e información financiera se lleve a cabo tomando como referentes las Normas Internacionales de Información Financiera NIIF junto con sus interpretaciones y el marco de referencia conceptual emitidas por el Consejo de Normas Internacionales de Contabilidad, International Accounting Standards Board, IASB por sus siglas en inglés y establece que se hará una adopción por endoso como en la comunidad Europea de acuerdo a 3 grupos, grupo 1 conformado por las compañías de interés público, grupo 2 conformado por las por las grandes empresas y medianas, grupo 3 las Pymes.
De acuerdo a esta clasificación las empresas del grupo 1 aplicaran las NIIF plenas, grupo 2 se les aplicara NIIF Pymes o si lo desean podrán aceptar NIIF plenas y grupo 3 se emitirá un plan de contabilidad para Pymes.

Que International Financial Reporting Standards, IFRSen el mes de noviembre de 2011 anunció la publicación de la traducción al español de las Normas Internacionales de Información Financiera que corresponden al texto utilizado para la aplicación de las Normas Internacionales de Información Financiera NIIF, que no incluyen el material complementario tal como los Fundamentos de las Conclusiones y la Guía de Implementación. La traducción al español de las Normas Internacionales de Información Financiera NIIF vigentes a11 ${ }^{\circ}$ de enero de 2011 se encuentra disponible en la página Web "Official IFRS Translations".

Con el objeto de avanzar en el proceso de convergencia hacia los estándares internacionales de aceptación mundial en materia de información financiera de que trata la Ley 1314 de 2009, es necesario conocer los impactos que conlleva este cambio fundamental en los procesos contables y de divulgación de información empresarial en Colombia, por lo cual se establece un ejercicio de aplicación voluntaria de las Normas Internacionales de Información Financiera NIIF, los cuales serán analizados por las diferentes autoridades únicamente con el propósito de medir si tales estándares internacionales resultan eficaces o apropiados para los entes en Colombia y de determinar las medidas regulatorias que deberán adoptarse, de tal manera que al terminar este ejercicio las obligaciones que se establezcan resulten razonables y acordes a la realidad económica del país. 
Para reglamentar la ley 1314 del 2009 el gobierno emitió los decretos 2784 del 28 de Diciembre del 2012 y el 2706 del 27 de Diciembre del 2012 en el primero se establece el marco técnico y normativo así como el cronograma de aplicación del mismo para las empresas que conforman el grupo 1 indicando quienes conforman el grupo 1 así como el periodo de preparación obligatoria, un periodo de transición o de apertura el cual inicio el 1 de Enero del 2014, una fecha de aplicación 31 de Diciembre del 2014 y fecha de reportes de Estados Financieros a 31 de Diciembre del 2015. Este primer grupo es el que utiliza NIIF plenas (Emisores de valores, entidades de interés público, más de doscientos (200) trabajadores, subordinada o sucursal que aplica NIIF plenas, matriz o asociada que aplicara NIIF plenas, importa o exporta más del 50\% de compra/venta)

El segundo decreto establece el marco técnico normativo de información financiera para las microempresas, el cual comprende el cronograma de aplicación igual al de las empresas del grupo 1 y el marco técnico normativo de información financiera para las microempresas, (Son aplicables para aquellas empresas que tienen máximo diez (10) trabajadores, activos inferiores a quinientos (500) SMLV, régimen simplificado de IVA, ingresos inferiores a 6.000 SMLV)

El 14 de Marzo del 2012 con base al documento de direccionamiento del Estratégico del Consejo Técnico de la Contaduría Pública, la Superintendencia de Sociedades emite la circular externa 115-000002 sobre el proceso de convergencia de las normas de contabilidad e información financiera con estándares internacionales NIC_NIIF imparte instrucciones para facilitar la aplicación del decreto 3022 de Diciembre 27 del 2013 en el cual se establece las sociedades que conforman el grupo 2. En el artículo
3 de este decreto se indica el cronograma de aplicación NIC_NIIF para las sociedades que conforman el grupo 2 y quienes deben aplicar NIIF Pymes

Las pequeñas y medianas empresas las denominadas Pymes se describe tomando como base la propuesta del Consejo Técnico de la Contaduría Pública en su documento de Direccionamiento Estratégico, se encuentra dos definiciones de una Pyme recogida por la NIIF para Pyme y la definición legal de las Pyme (Ley 590 de 2000, modificada por la Ley 905 de 2004).

Para la NIIF para Pyme, las Pyme son aquellas que no tienen obligación pública de rendir cuentas pero presentan estados financieros de propósito general para usuarios externos.

Se considera que una entidad no tiene la obligación pública de rendir cuentas:

a. Sus instrumentos de deuda o de patrimonio se negocian en un mercado público o están en proceso de emitir estos instrumentos para negociarse en un mercado público (ya sea una bolsa de valores nacional o extranjera, o un mercado fuera de la bolsa de valores, incluyendo mercados locales o regionales), o

b. Una de sus principales actividades es mantener activos en calidad de fiduciaria para un amplio grupo de terceros.

Este suele ser el caso de los bancos, las cooperativas de crédito, las compañías de seguros, los intermediarios de bolsa, los fondos de inversión y los bancos de inversión. La otra definición propone que una Pyme es aquella que responda a dos (2) de los siguientes parámetros: 
Pequeña Empresa

a. Planta de personal entre once (11) y cincuenta (50) trabajadores.

b. Activos totales por valor entre quinientos uno (501) y menos de cinco mil (5.000) salarios mínimos mensuales legales vigentes.

\section{Mediana Empresa}

a. Planta de personal entre cincuenta y uno (51) y doscientos (200) trabajadores.

b. Activos totales por valor entre cinco mil uno (5.001) a treinta mil (30.000) salarios mínimos mensuales legales vigentes.

Tabla 2. Cronograma de aplicación de NIIF para Pymes en Colombia

\begin{tabular}{|c|c|c|}
\hline No. & ACTIVIDADES & FECHA \\
\hline 1 & $\begin{array}{c}\text { Periodo de } \\
\text { preparación } \\
\text { obligatoria }\end{array}$ & $\begin{array}{c}\text { Enero 01 a } \\
\text { diciembre 31 } \\
\text { de 2014 }\end{array}$ \\
\hline 2 & Fecha de transición & $\begin{array}{c}\text { Enero 01 de } \\
2015\end{array}$ \\
\hline 3 & $\begin{array}{c}\text { Estado de situación de } \\
\text { apertura }\end{array}$ & $\begin{array}{c}\text { Enero 01 de } \\
2015\end{array}$ \\
\hline 4 & $\begin{array}{c}\text { Periodo de transición } \\
5\end{array}$ & $\begin{array}{c}\text { Enero 01 a 31 } \\
\text { de diciembre } \\
\text { de 2015 }\end{array}$ \\
\hline 6 & $\begin{array}{c}\text { Fechana de aplicación } \\
\text { NIIF }\end{array}$ & $\begin{array}{c}\text { Enero } 01 \text { de } \\
2016\end{array}$ \\
\hline 7 & $\begin{array}{c}\text { Primer periodo de } \\
\text { aplicación }\end{array}$ & $\begin{array}{c}\text { Enero } 01 \text { al 31 } \\
\text { de diciembre } \\
\text { de 2016 }\end{array}$ \\
\hline 8 & $\begin{array}{c}\text { Fecha reporte estados } \\
\text { financieros NIIF }\end{array}$ & $\begin{array}{c}\text { Diciembre } 31 \\
\text { de 2016 }\end{array}$ \\
\hline
\end{tabular}

Fuente: Accounter (2012).

El 14 de marzo del 2013 La Superintendencia de Sociedades emite la circular 115-000003 sobre el proceso de implementación del marco Técnico Normativo de Información Financiera para las microempresas y plan de Implementación acorde al decreto 2706 de Diciembre 27 del 2012.
Es asi como en Colombia para el año 2016 todas las empresas deberán presentar sus estados financieros de acuerdo a las normas internacionales vigentes. Heidrich (2011) enumeró algunos obstáculos que afronta Latinoamérica frente al tema de la adopción NIIF:

- Los recursos humanos disponibles en el mercado son limitados, hay pocos profesionales capacitados en el medio sobre este tema.

- Los entrenamientos para los profesionales disponibles en el mercado son limitados y normalmente no cumplen con la profundidad que las compañías necesitan.

- Aumento en los costos por conceptos de asesorías y procesos de implementación y conversión a NIIF.

¿Estamos preparados?

\section{REFERENCIAS BIBLIOGRÁFICAS}

Apaza Meza, Mario (2008): "El plan general de contabilidad para Pymes en España”, Revista legis de contabilidad y auditoría, número 36.

Actualicese.com (2005). Normas Internacionales (NICs) Vigentes. Septiembre 21 de 2005. Recuperado el 24 de abril de 2014. http:// actualicese.com/actualidad/2005/09/21/ normas-internacionales-vigentes/.

Accounter (2012). Nuevo cronograma oficial aplicación NIIF en Colombia. Septiembre de 2012. Recuperado el 25 de abril de 2014. http://accounter.co/boletines/14343-nuevo-cronograma-oficial-aplicacion-niif-en-colombia.html.

ACFÍ Auditores, Contadores y Consultores Financieros. (s/f). ¿Qué son las NIIF y cuántos hay? Recuperado el 22 de abril de 2014. http://www.auditoresycontadores.com/ articulos/contabilidad/niif/48-ique-sonlas-niif-y-cuantos-hay. 
Blanco, Yanel (1997). Normas internacionales de Contabilidad. Editorial Roesga.

Cañibano, L., y Tua Pereda, J. (1985). "Naturaleza y Filosofía de los Principio Contables". En Revista Española de Financiación y Contabilidad. núm. 47

Consejo Técnico de la Contaduría Pública. Análisis de la implantación de estándares de contabilidad y auditoría en empresas de interés público en Colombia. Divergencia entre la normativa del IASB y la normativa contable colombina. En Revista Internacional LEGIS Contabilidad \&Auditoria. No.27 Julio-Septiembre del 2006.

Endress Gómez, Sergio (2010), Revista legis de contabilidad y auditoría, número 43 , publicación Julio- Septiembre de 2010.

Edgar Emilio Salazar-Baquero (compilador) (2009). Bogotá Colombia. Por profesores del área de Contabilidad Financiera, departamento de Ciencias Contables. Universidad Javeriana. Julio-diciembre 2009.

Franco, Rafael (2002). ¿Adaptar, adoptar o armonizar? el gran dilema. Ámbito Jurídico. Pág. 5.

Gonzalo, J. (2011.Colombia, ¿mal preparada para adoptar las NIIF?, 10 de marzo de 2011. Recuperado 7 Mayo 2014 http://actualicese.com/actualidad/2011/03/10/colombia-mal-preparada-para-adoptar-las-niif/.

Gil, Jorge (2009). “Adopción de las normas internacionales de información financiera (NIIF/ IFRS) en América Latina: el plan de argentina" en revista legis de contabilidad y auditoría. Número 38, 15-64.

Internacional Accounting Standars Board (2005). "La Influencia de los grupos de interés en el proceso normativo del Internacional Accounting Standars Board: el caso de la NIIF para Pymes"; tesis doctoral; Rafael Jesús Bautista Mesa.

Montesinos Julve, V. (1978), Formación histórica, corriente doctrinales y programa de investigación de la Contabilidad, en Revista Técnica Contable, vol. 30, 353.
Montesinos Julve, V. (1994). Recopilación de artículos publicados en la Revista Técnica Contable (España), efectuada en una publicación de la Cátedra Contabilidad Superior de la Facultad de Ciencias Económicas de la Universidad de Buenos Aires.

Null Value, 31 de Julio de 2007 artículo publicado por portafolio.com.co, sección de economía.

Ramírez H. S. L Guía NIIF para Pymes Recuperado 30 de Abril 2014. http://www.unilibrecali.edu.co/facultadadministracion/ images/stories/PDF_noticias/guia_niif_ para\%20Pymes.pdf

Rodríguez Astrid Genoveva, Gonzales Beltrán Andrea Carolina (2003). "La realidad de la Pyme colombiana, desafío para el desarrollo". FUNDES Colombia. (Septiembre del 2003).

Tua Pereda, J. (1983) Principios y Normas de contabilidad. Historia, Metodología y entorno de la regulación contable, Instituto de Planificación Contable, Madrid.

Tua Pereda, J. (1988). Evolución de la contabilidad a través de sus conceptos. En Revista Contaduría Universidad de Antioquia" núm. 13 CFACES, Dpto. de Contaduría, Medellín.

Tua Pereda (1990). Algunas implicaciones del paradigma de utilidad en la disciplina contable. Revista Contaduría Universidad de Antioquia. núm. 16.

Tua Pereda, J. (1991). La Investigación Empírica en Contabilidad. La hipótesis de eficiencia del mercado. Instituto de Contabilidad y Auditoría de cuentas, Ministerio de Economía y Hacienda, Madrid.

Tua Pereda, J. (2004). AECA: El marco conceptual, soporte de las Normas Internacionales. Revista de la Asociación Española de Contabilidad y Administración de empresas, No. 66, 4-10.

Tua Pereda, J. (1995a). Evolución del concepto de contabilidad a través de sus definiciones. Revista Contaduría. Universidad de Antioquia, núm. 13. 
Tua, Pereda, J. (2004). Evolución y situación actual del pensamiento contable, en Revista internacional de Contaduría y Auditoria núm. 20.

Zhang, Silin (2010). "La importancia de las Pymes para chile y china" Biblioteca del congreso Nacional de Chile. Caso colombiano publicado el 14 de Octubre de 2010.

Zeff, S. (2012). La Evolución del IASC, al IASB, y los retos que enfrenta. Contaduría Universidad de Antioquia, 60, 119-164.

\section{ROCÍO VERGARA-DE LA OSSA}

Contaduría Pública de la Universidad Jorge Isaza Cadavid. Especialista en Gerencia de Impuestos de la Universidad Externado de Colombia. Magíster en Educación de la Universidad Simón Bolívar- Sede Barranquilla. Cuenta con más de 20 años de experiencia en el sector productivo en empresas de la ciudad de Cartagena. Actualmente se desempeña como Directora de los Programas Tecnología en Contabilidad Sistematizada y Contaduría Pública de la Fundación Universitaria Antonio de Arévalo.

\section{CLAUDIA INÉS LONDOÑO-VEGA}

Magister en Educación Basada en Competencias de la Universidad del Valle de México. Contador Público de la Universidad de Cartagena. Especialista en Revisoría Fiscal y Contraloría de la Corporación Universitaria Remington. Jefe de Planeación de la Base Naval ARC "Bolívar". Actualmente se desempeña como docente de medio tiempo en la Fundación Tecnológica Antonio de Arévalo.

\section{NAZLY DEL CARMEN PÉREZ-BENÍTEZ}

Economista de la Universidad de Cartagena y Contador Público de la Corporación Universitaria Rafaél Núñez. Especialista en Gerencia Financiera de la Universidad Jorge Tadeo Lozano. Magister en Dirección de Empresas. Actualmente de desempeña como docente de la Fundación Tecnológica Antonio de Arévalo.

\section{ROBERTO TORRES-CASTELLAR}

Doctorante en Ciencias Económicas de la Universidad del Zulia. MBA - Magíster en administración y Especialista en Gerencia del Talento Humano de la Universidad Tecnológica de Bolívar. Economista de la Universidad de Cartagena. Actualmente se desempeña como Coordinador de investigación de la Fundación Tecnológica Antonio de Arévalo.

\section{Para citaciones:}

Vergara-De La Ossa, R., Londoño-Vega, C., Pérez-Benítez, N., \& Torres-Castellar, R. (2015). La adopción de las normas internacionales de información financiera en Colombia. Panorama Económico, 23, 119-132.

Recepción del artículo: 5 de Marzo de 2015 Concepto de evaluación: 23 de Junio de 2015 Aceptación del artículo: 18 de Julio de 2015 